\title{
Dominance of an alternative CLIP sequence in the celiac disease associated HLA-DQ2 molecule
}

\author{
Martina Wiesner • Dariusz Stepniak • \\ Arnoud H. de Ru • Antonis K. Moustakis • \\ Jan W. Drijfhout • George K. Papadopoulos • \\ Peter A. van Veelen • Frits Koning
}

Received: 5 March 2008 / Accepted: 26 May 2008/Published online: 27 June 2008

(C) The Author(s) 2008

\begin{abstract}
During assembly, HLA class II molecules associate with the invariant chain. As the result, the peptide-binding groove is occupied by an invariant chain peptide termed CLIP (class-II-associated invariant chain peptide; sequence MRMATPLLM). By mass spectrometry, we have now characterized peptides that are naturally present in HLADQ2. This analysis revealed that 22 variants of Ii-derived peptides are associated with HLA-DQ2. Strikingly, the large majority of those do not contain the conventional CLIP sequence MRMATPLLM, but instead a peptide that partially overlaps with CLIP, sequence TPLLMQALPM. Peptide binding studies indicate that this alternative CLIP peptide has superior HLA-DQ2 binding properties compared to the conventional CLIP and that the minimal nine-amino-acid binding core consists of the sequence PLLMQALPM, findings that could be corroborated by molecular simulation. The alternative CLIP peptide was also found to be present in
\end{abstract}

M. Wiesner · D. Stepniak · A. H. de Ru - J. W. Drijfhout

P. A. van Veelen $\cdot$ F. Koning $(\varangle)$

Department of Immunohematology and Blood Transfusion,

Leiden University Medical Centre, E3-Q,

P.O. Box 9600, 2300 RC Leiden, The Netherlands

e-mail: f.koning@lumc.nl

A. K. Moustakis

Department of Organic Farming,

Technology Educational Institute of Ionian Islands,

29100 Argostoli, Cephallonia, Greece

G. K. Papadopoulos

Laboratory of Biochemistry and Biophysics,

Faculty of Agricultural Technology, Epirus Institute of Technology,

47100 Arta, Greece

D. Stepniak

La Jolla Institute of Allergy and Immunology,

La Jolla, CA 92037, USA
HLA-DQ2 molecules isolated from human thymus. Moreover, the alternative CLIP peptide was also found in association with HLA-DQ8. Together, these results indicate that HLA-DQ2 and HLA-DQ8 associate with an alternative CLIP sequence, a property that may relate to the strong association between HLA-DQ molecules and human autoimmune diseases.

Keywords HLA class II · HLA-DQ2 - CLIP.

Alternative binding
Abbreviations
Ii invariant chain
CLIP class-II-associated invariant chain peptide

\section{Introduction}

The MHC class II $\alpha$ and $\beta$ subunits associate with an invariant chain (Ii) trimer shortly after biosynthesis in the endoplasmic reticulum, generating a stable complex (Roche et al. 1991). Part of the Ii-chain, commonly referred to as CLIP (class-II-associated invariant $c$ hain peptide), occupies the peptide-binding groove of MHC class II molecules, thus preventing peptide loading in the endoplasmatic reticulum (Bakke and Dobberstein 1990). Moreover, Ii facilitates the transport of the MHC class II molecules to the endosomal compartment where Ii is degraded by proteases. Subsequently, CLIP is released from the groove by the catalytic action of HLA-DM and exchanged for immunogenic peptides derived from proteins that have entered the endosomal/phagolysosomal system (Rocha and Neefjes 2008). Several autoimmune diseases, including type I diabetes and celiac disease, are HLA-associated. 
In a recent study, we have reported the characterization of a large number of naturally HLA-DQ2-bound peptides through mass spectrometry (Stepniak et al. 2008). In agreement with two previous studies, the most abundant peptide identified was the HLA-class-I-derived peptide IEQEGPEYW (van de Wal et al. 1996, Vartdal et al. 1996). Similarly, the second most abundant peptide was found to be invariant chain (li)-derived (van de Wal et al. 1996; Vartdal et al. 1996). We estimate that at least $10 \%$ of all HLA-DQ2 molecules is occupied by an invariant-chainderived peptide. Twenty-two invariant-chain-derived peptides were identified, and a minimal core of ten amino acids was found to be present in all length variants [sequence TPLLMQALPM (CLIP 94-103); Fig. 1a]. Surprisingly, the sequence TPLLMQALPM was distinct from the CLIP peptide MRMATPLLM (CLIP 90-98) found to occupy the binding groove of all other human and murine class II molecules investigated (Chapman 1998). To determine the ratio between the CLIP and the alternative CLIP peptide in HLA-DQ2, we determined the relative abundance of the length variants of the Ii-derived peptides by mass spectrometry. This was done by integration of selected peaks in the mass chromatograms, done under the assumption that the relative response is similar for these species, which is appropriate since these species are length variants. The vast majority (approximately 90\%) of the Ii-derived peptides contained the alternative CLIP sequence, while $10 \%$ contained the CLIP sequence (Fig. 1a).

To test if the alternative CLIP peptide is also present in HLA-DQ2 molecules expressed in the human thymus, we affinity purified HLA-DQ2 molecules from the thymus of two approximately 1.5-year-old HLA-DQ2-positive children. Subsequently, the bound peptides were eluted and characterized by mass spectrometry. A single Ii-derived peptide was identified which contained the CLIP sequence: TPLLMQALMGALPQ (CLIP 94-107; Fig. 1b). This peptide is identical to one of the most abundant peptides found in HLA-DQ2 molecules purified from an EpsteinBarr virus (EBV)-transformed cell line (Fig. 1a). Together, this indicates that also in the thymus, the alternative CLIP peptide is present in HLA-DQ2.

Two peptides, containing either CLIP (MRMATPLLM QAL; CLIP 90-101) or the alternative sequence (ATPL LMQALPMGALP; CLIP 93-107), were tested for their capacity to bind to HLA-DQ2 in a cell-free competition assay (Fig. 2a). The results showed a significantly lower $\mathrm{IC}_{50}$ value for the alternative CLIP sequence ATPLLM QALMGALP (CLIP 93-107), indicating a higher binding affinity. We further tested two nonamers containing CLIP (MRMATPLLM; CLIP 90-98) and the predicted binding frame of the alternative CLIP (PLLMQALPM: termed DQ2-CLIP hereafter; Fig. 2a) again showing a fourfold lower $\mathrm{IC}_{50}$ for DQ2-CLIP.
To test the predicted binding frame of the DQ2-CLIP sequence, we next analyzed the binding of $\mathrm{N}$ - and $\mathrm{C}$ terminally truncated length variants of CLIP 92-107 (MATPLLMQALPMGALP; Fig. 2b). Deletion of $\mathrm{P}_{95}$ and $\mathrm{M}_{103}$ was found to abrogate binding almost completely, indicating PLLMQALPM (CLIP 95-103) as the nineamino-acid binding core.

To study the binding of DQ2-CLIP in more detail, we next determined which amino acids function as anchor residues in PLLMQALPM (CLIP 95-103). For this purpose, the impact of systematic substitution of amino acids in PLLMQALPM by lysine (K) was determined (Fig. 2c). Most dramatic effects could be seen for substitutions at positions $\mathrm{p} 1, \mathrm{p} 4, \mathrm{p} 6$, and $\mathrm{p} 9$, known to be important anchor positions where no positive charges are allowed. In addition, strong effects could also be seen at the p7 anchor position and the non-anchor position p8. This latter observation may reflect the preference of HLA-DQ2 for a proline at the $\mathrm{p} 8$ position (Stepniak et al. 2008).

Additionally, modeling of HLA-DQ2 complexed with the peptide PLLMQALPM showed that it fits well in the groove of HLA-DQ2 (Fig. 3a and b). Moreover, it is predicted to bind better than the conventional CLIP peptide MRMATPLLM (unpublished data), a prediction that is confirmed by the observed superior binding of DQ2-CLIP (Fig. 2a).

Since the spacing of the anchor residues in DQ2-CLIP is identical to that of the dominant HLA-class-I-derived peptide IEQEGPEYW (van de Wal et al. 1996; italicized amino acids are residues at anchor positions) as well as gluten-derived $\mathrm{T}$ cell stimulatory peptides (Vader et al. 2003), we conclude that the DQ2-CLIP peptide binds to HLA-DQ2 molecules in a similar binding register. In conclusion, the minimal core sequence within the DQ2CLIP peptide that binds strongly to HLA-DQ2 is PLLMQALPM (residues 95-103) in which the italicized amino acids are residues at anchor positions.

Because celiac disease and type I diabetes are also associated with HLA-DQ8, we also analyzed naturally HLA-DQ8-bound peptides through mass spectrometry. The invariant chain was represented again by 22 peptides with a minimal core of ten amino acids present in all length variants, which is identical to that found in HLA-DQ2 [sequence TPLLMQALPM (CLIP 94-103); data not shown]. In adidtion, binding studies revealed that the peptide ATPLLMQALPMGALP (CLIP 93-107) binds slightly better than the peptide MRMATPLLMQAL (CLIP 90-101; data not shown). These results thus indicate that next to HLA-DQ2, HLA-DQ8 also appears to harbor an alternative CLIP peptide. Further studies will be required to determine the exact binding frame of this alternative CLIP peptide in HLA-DQ8.

It is now widely accepted that positive selection in the thymus is promiscuous (Ignatowicz et al. 1996), that is, a 
Fig. 1 Identification of an alternative CLIP peptide in HLA-DQ2. A Alignment of the length variants of the invariantchain-derived peptides present in HLA-DQ2 expressed on EBV-transformed B cells. The solid line marks the predicted alternative CLIP sequence specific for HLA-DQ2; the dashed line marks the CLIP sequence found in other HLA class II molecules. B Tandem mass spectrum of one of DQ2-CLIP peptides. The amino acid sequence of the peptide is indicated on top of the spectrum, including the expected $b$ - and $\mathrm{y}$-fragment ions. Observed fragment ions are underlined and indicated in the spectrum. The correct identity of the peptide was proven by tandem mass spectrometry of the synthetic compound a

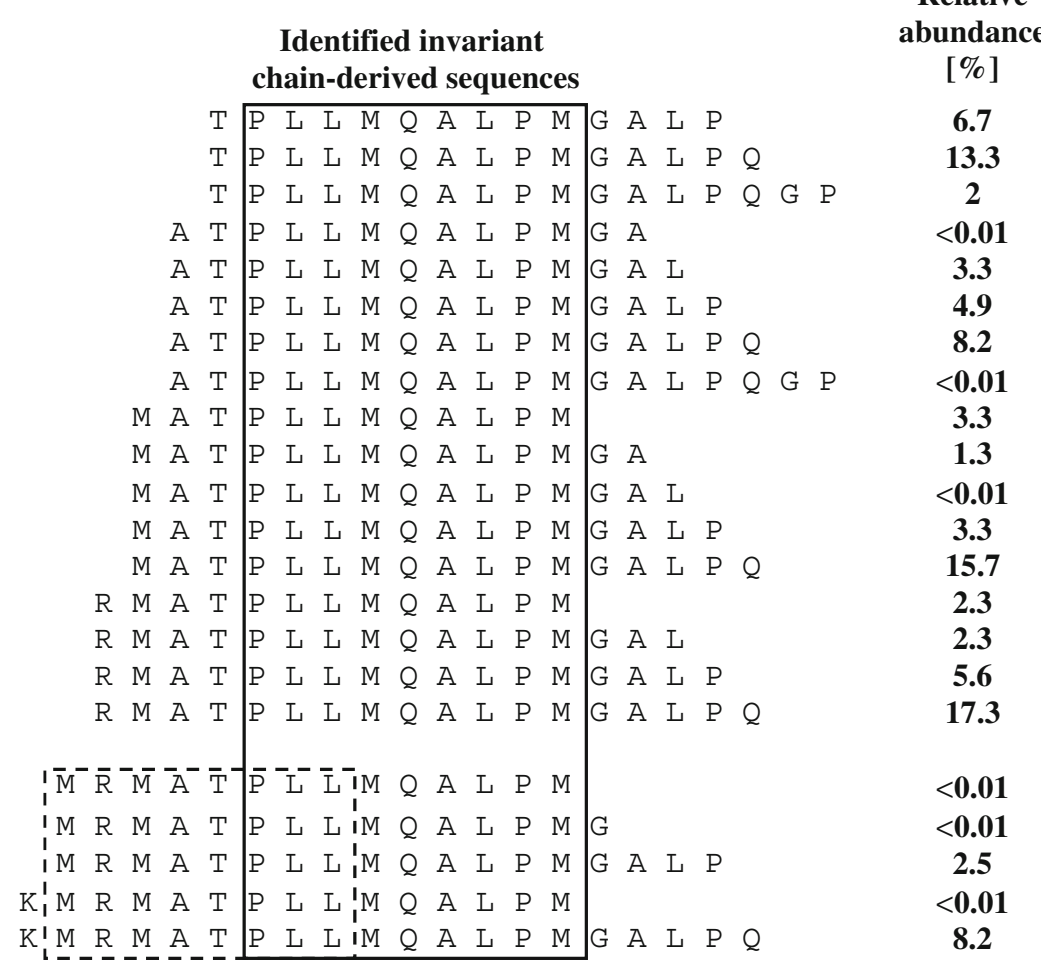

b single self-peptide is able to mediate positive selection of large numbers of $\mathrm{T}$ cells with the ability to respond to various peptide antigens (Ignatowicz et al. 1997). It has also been demonstrated that in mice lacking DM molecules required for the dislocation of CLIP from MHC class II

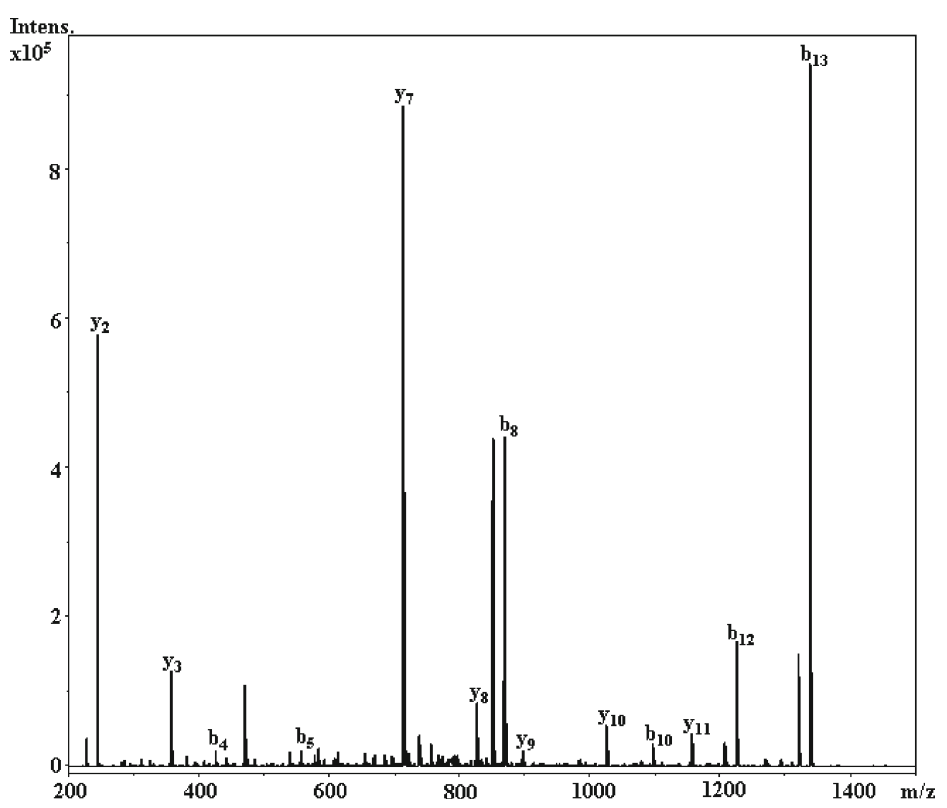

(Fukui et al. 1997; Martin et al. 1996), 15-50\% of the normal number of $\mathrm{CD}^{+}{ }^{+} \mathrm{T}$ cells are selected. Moreover, the $\mathrm{T}$ cell receptor repertoire in these mice was different from that in wild-type mice, suggesting that MHC class II-CLIP complexes can select a large and diverse repertoire of $\mathrm{T}$ 
a

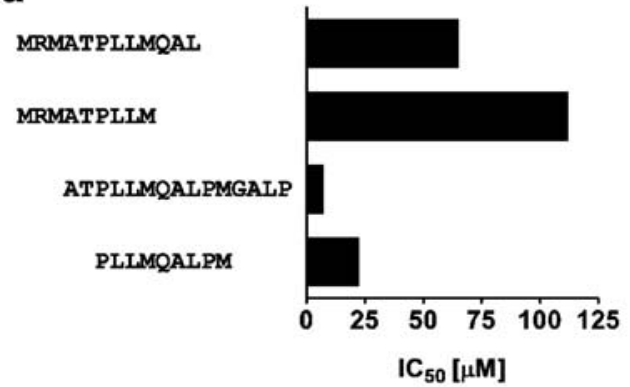

b

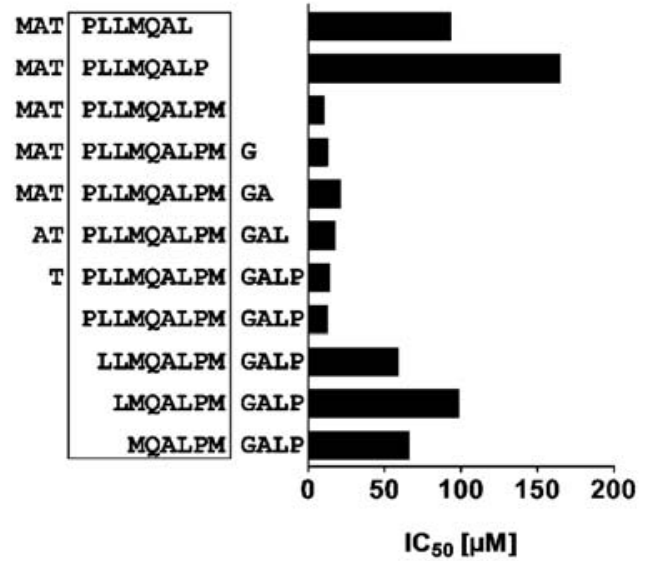

C

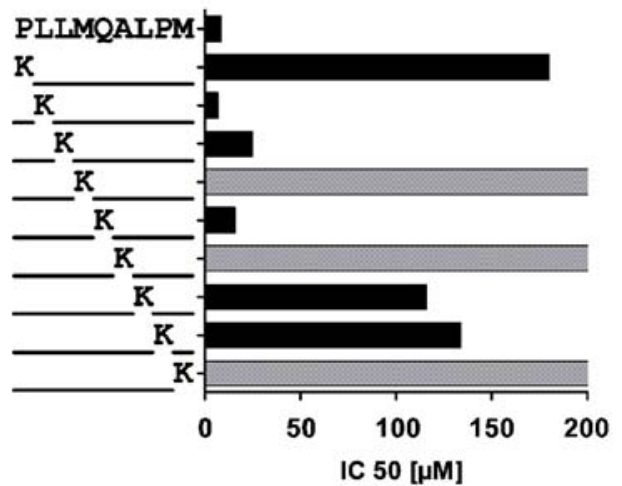

Fig. 2 Identification of an alternative CLIP sequence. A Binding of two versions of the CLIP peptide to HLA-DQ2 and the binding of the predicted minimal core of DQ2-CLIP (PLLMQALPM). B Binding of a series of truncated versions of CLIP $90-105$ to HLA-DQ2. C Binding of a series of CLIP 93-101 lysine substitution analogues. Gray columns indicate an $\mathrm{IC}_{50}$ above $200 \mu \mathrm{M}$ representing weak or no binding. Each peptide was tested in at least two independent experiments; representative results are shown

cells. Given the dominant presence of DQ2-CLIP in HLADQ2, it is therefore possible that a substantial proportion of HLA-DQ2-restricted $\mathrm{T}$ cells in the periphery has been selected by HLA-DQ2-[DQ2-CLIP] complexes in the thymus. As HLA-DQ8 also appears to harbor an alternative CLIP peptide, this may similarly apply to $\mathrm{T}$ cell receptor selection in HLA-DQ8 individuals. It is tempting to speculate that this relates to the fact that HLA-DQ2 and
HLA-DQ8 confer susceptibility for the common autoimmune diseases celiac disease and type I diabetes (Nepom and Kwok 1998; Todd et al. 1987).

\section{Concluding remarks}

In conclusion, we have observed that HLA-DQ2 molecules preferentially associate with an alternative form of the invariant-chain-derived CLIP peptide, DQ2-CLIP. The DQ2-CLIP has an at least four times higher binding affinity to HLA-DQ2 than the previously described classical CLIP sequence. Similarly, we observed that HLA-DQ8 harbors an alternative CLIP peptide. This property may relate to the strong association between these HLA-DQ molecules and human autoimmune diseases.

a

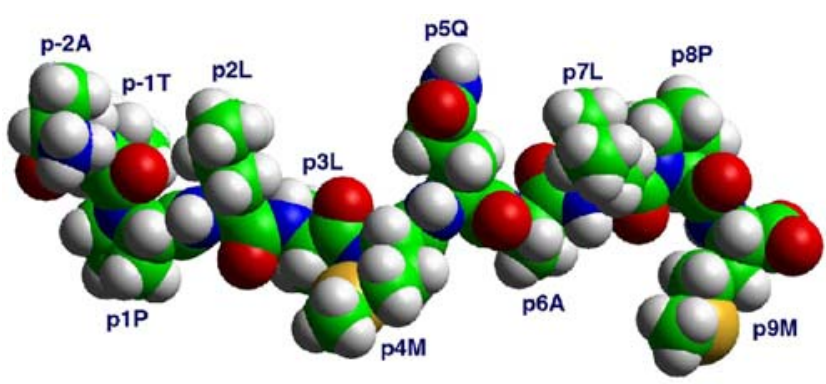

$\mathrm{b}$

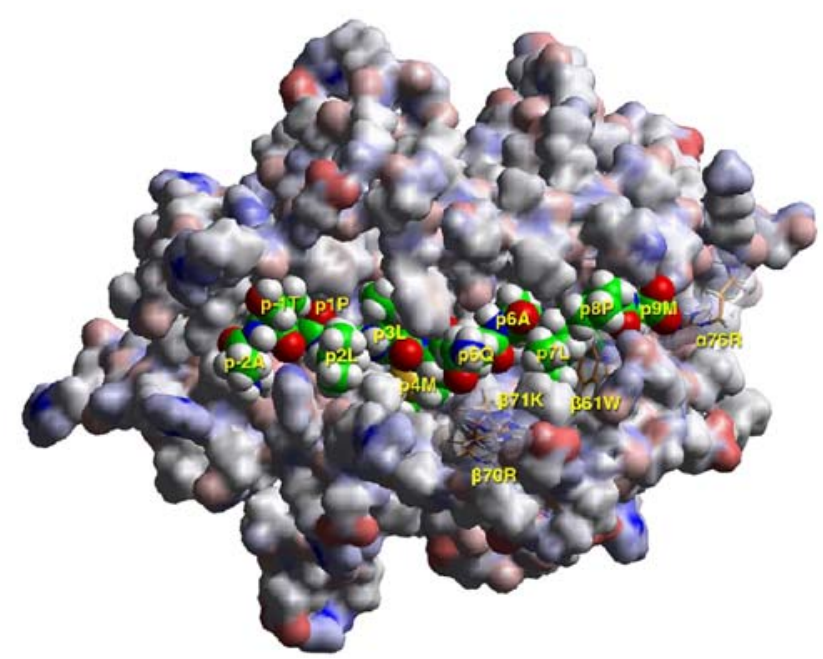

Fig. 3 Computer modeling of DQ2-CLIP in association with HLADQ2 based on the known DQ2-structure. A Side view of the DQ2CLIP peptide (Ii 94-104) as it appears in complex with HLA-DQ2. B Shown is the TCR view of HLA-DQ2 $(\mathrm{A} 1 * 0501 / \mathrm{B} 1 * 0201)$ in complex with the DQ2-CLIP peptide (94-104 of Ii; core nonamer 96-104). The HLA-DQ2 molecule is shown in a van der Waals surface representation color coded according to the atomic charges (positive, blue; negative, red; neutral, gray); the surfaces for four residues $(\alpha 76 \mathrm{Arg}, \beta 61 \mathrm{Trp}, \beta 70 \mathrm{Arg}, \beta 71 \mathrm{Lys})$ are transparent so that the respective residues can all be made visible. The peptide is shown in space filling mode (atomic color code: carbon, green; oxygen, red; nitrogen, blue; hydrogen, white; sulfur, yellow) 
Acknowledgments This research has been supported by a Marie Curie Fellowship of the European Community programme Drugs for Therapy under the contract number MRTN-CT-2004-512385 and the Celiac Disease Consortium, an Innovative Cluster approved by the Netherlands Genomics Initiative, and partially funded by the Dutch Government (BSIK03009). The authors thank B.O. Roep and J. van Bergen for critically reading the manuscript.

Open Access This article is distributed under the terms of the Creative Commons Attribution Noncommercial License which permits any noncommercial use, distribution, and reproduction in any medium, provided the original author(s) and source are credited.

\section{References}

Bakke O, Dobberstein B (1990) MHC class II-associated invariant chain contains a sorting signal for endosomal compartments. Cell 63:707-716

Chapman HA (1998) Endosomal proteolysis and MHC class II function. Curr Opin Immunol 10:93-102

Fukui Y, Ishimoto T, Utsuyama M, Gyotoku T, Koga T, Nakao K, Hirokawa K, Katsuki M, Sasazuki T (1997) Positive and negative $\mathrm{CD}^{+}$thymocyte selection by a single MHC class II/peptide ligand affected by its expression level in the thymus. Immunity 6:401-410

Ignatowicz L, Kappler J, Marrack P (1996) The repertoire of T cells shaped by a single MHC/peptide ligand. Cell 84:521-529

Ignatowicz L, Rees W, Pacholczyk R, Ignatowicz H, Kushnir E, Kappler J, Marrack P (1997) T cells can be activated by peptides that are unrelated in sequence to their selecting peptide. Immunity 7:179-186
Martin WD, Hicks GG, Mendiratta SK, Leva HI, Ruley HE, Van Kaer L (1996) H2-M mutant mice are defective in the peptide loading of class II molecules, antigen presentation, and $\mathrm{T}$ cell repertoire selection. Cell 84:543-550

Nepom GT, Kwok WW (1998) Molecular basis for HLA-DQ associations with IDDM. Diabetes 47:1177-1184

Rocha N, Neefjes J (2008) MHC class II molecules on the move for successful antigen presentation. EMBO J 27:1-5

Roche PA, Marks MS, Cresswell P (1991) Formation of a ninesubunit complex by HLA class II glycoproteins and the invariant chain. Nature 354:392-394

Stepniak D, Wiesner M, de Ru AH, Moustakas AK, Drijfhout JW, Papadopoulos GK, van Veelen PA, Koning F (2008) Large-scale characterization of natural ligands explains the unique glutenbinding properties of HLA-DQ2. J Immunol 180:3268-3278

Todd JA, Bell JI, McDevitt HO (1987) HLA-DQ beta gene contributes to susceptibility and resistance to insulin-dependent diabetes mellitus. Nature 329:599-604

Vader W, Stepniak D, Kooy Y, Mearin ML, Thompson A, Spaenij L, Koning F (2003) The HLA-DQ2 gene dose effect in Celiac Disease is directly related to the magnitude and breadth of gluten-specific T-cell respnoses. Proc Natl Acad Sci USA 100:12390-12395

van de Wal Y, Kooy YMC, Drijfhout JW, Amons R, Koning F (1996) Peptide binding characteristics of the coeliac disease-associated DQ(alpha $1 * 0501$, beta $1 * 0201)$ molecule. Immunogenetics 44:246-253

Vartdal F, Johansen BH, Friede T, Thorpe CJ, Stevanovic S, Eriksen JE, Sletten K, Thorsby E, Rammensee HG, Sollid LM (1996) The peptide binding motif of the disease associated HLA-DQ (alpha $1 * 0501$, beta $1 * 0201$ ) molecule. Eur J Immunol 26:2764 2772 